\title{
TRIBUN UND PROPHET
}

\author{
MOSES IESS ALS PARISER KORRESPONDENT
}

DER ILLINOIS STAATS-ZEITUNG

I.

Wer das heutige Chikago mit seinem im Hintergrund bleibenden deutschen Element durchstreift, vermag sich kaum vorzustellen, dass es einmal einige zwanzig Prozent der Einwohnerschaft bildete. ${ }^{1}$ Nicht bloss die Zahlenstärke, auch die Geschlossenheit der aus Deutschland Stammenden beeindruckt den rückblickenden Beobachter. Angehörige aller Religionen fanden sich damals zu ausgesprochen prodeutschen Kundgebungen zusammen ${ }^{2}$, besassen doch Katholizismus, Protestantismus und Judentum in den Worten eines massgeblichen Führers „nicht mehr Bedeutung als schwarze, braune, blonde oder rothe Haare". ${ }^{3}$ Kein Wunder also, dass die Vorstellung entstehen konnte, diese Stadt sei ,zum grossen Theile eine deutsche Schöpfung".4

1 Der Schwund der deutschen Kultur ist ein noch ungenügend beleuchtetes Problem, und nicht allein für die USA. Wertvolle örtliche Einblicke bieten Dieter Cunz, Egg Harbor City: New Germany in New Jersey, Society for the History of the Germans in Maryland, Twenty-ninth report, Baltimore I956, S. 9-29 und Henry Pelling, The rise and decline of socialism in Milwaukee, Bulletin of the International Institute of Social History, I955, Nr. 2, S. 9r-103.

2 Siche Chicago und sein Deutschthum, hrsg. von der German-American Biographical Pub. Co., Cleveland, I 901-1902, namentlich S. I 27- I 28; Tagesausgabe der Illinois StaatsZeitung - hiernach zitiert als T.I.S.Z. - Bd. XXIII (1870), Nr. 173, S. 2 mit der Rede von Eduard Salomon; Nr. 179, S. 4, wo neben einer Notiz über den Deutschen Patriotischen Frauenverein ein längerer Sitzungsbericht der B'nai B'rith-Loge erscheint; Nr. 187, S. 2, wo eine gewisse jüdische Überempfindlichkeit besprochen wird, und Nr. 192, S. 2, wo verständliches Ressentiment und Identifizierung der Juden mit Deutschland behandelt sind.

${ }^{3}$ Hermann Raster an Bruno Bauer. Für die Benutzung des unveröffentlichten Briefs vom 12. Juli I 870 dankt der Verf. der Newberry-Bibliothek. Zu Bauer siehe Helmut Hirsch, Karl Friedricht Köppen, der intimste Berliner Freund Marxens, in: Denker und Kämpfer, Frankfurt I955, S. 19-8I und Hans-Joachim Schoeps, Hermann Wagener - ein konservativer Sozialist, in: Zeitschrift für Religions- und Geistesgeschichte, Bd. VIII (1956), S. 194 .

4 Wochenausgabe der Illinois Staats-Zeitung - hicrnach zitiert als W.I.S.Z. - Bd. XXIII (1869), Nr. 33, S. 3. 
Der das i 869 bei einem Sommernachts-Kommers vor 4000 Turnern verkündete, der Achtundvierziger Wilhelm Rapp ${ }^{1}$, wirkte leitend mit an einem Presserzeugnis, dessen Existenz die ehemalige Bedeutung des Chikagoer Deutschtums am besten illustriert. 1848, drei Jahre nach dem ersten deutschen Blatt Chikagos, dem „Volksfreund”, und wie dieses von dem Drucker Robert Bernhard Höffgen begründet, um „Bildung, Recht und Freiheit zu fördern” 2, erschien die „Illinois Staats-Zeitung” an dem dann „kaum noch auf den Titel Städtchen Anspruch habenden Platze in der Präirie" ${ }^{3}$ zunächst nur wöchentlich. Nach dem kurzfristigen Zwischenspiel zweier weiterer Herausgeber verhalf Hermann Kriege ${ }^{4}$ schon im folgenden Jahr der Publikation zum halbwöchentlichen und darauf dreimal wöchentlichen Erscheinen. ${ }^{5}$ Von 185 I an kam es zu einer Tagesausgabe. Jetzt rangierte über Höffgen der als Achtundvierziger ganz links, später zu Lincoln und der republikanischen Partei stehende Georg Schneider. ${ }^{6}$ Die Tageszeitung brachte es ihrerseits zu einem wöchentlichen Ableger. In der wohl hier nicht parteilichen Meinung Rapps hielt die StaatsZeitung nämlich in verschiedener Hinsicht den Vergleich mit den wichtigsten Organen der alten Heimat aus. ${ }^{7}$ Für europäische Leser wie für die ländlichen Bezieher der westlichen Staaten wurde daher aus den wesentlichen Beiträgen der täglichen Ausgabe eine mit der Post versandte Wochenausgabe zusammengestellt. Ihre Artikel fanden auf diese Weise mehr als nur lokale Verbreitung.

Unter den 280 deutschsprachigen Blättern, die es ehedem in den

1 Über diesen Redakteur, während dessen Amtszeit Moses Hess seine Mitarbeit aufnahm, unterrichten die sorgfältigen Angaben von Fritz Caspari, The Baltimore riots of 1861 , in: The Newberry Bulletin, second series, Nr. 9(1952), S. 274-283.

2 Aus Ein Abschiedsgruss, T.I.S.Z., Bd. XIV (186r), Nr. 238, S. 2.

${ }^{3}$ Ibid.

4 Wohl kein anderer wie der 1846 von Karl Marx als sentimentaler Kommunist verspottete New Yorker Publizist (siehe Maximilien Rubel, Bibliographie des Oeuvres de Karl Marx, Paris I956, S. 57). - Kriege gab vom Januar 1845 bis Dezember 1846 in Amerika den Volks-Tribun heraus. In einem Nekrologe (Republik der Arbeiter, I851, S. 15) schrieb Wilhelm Weitling, dass er ihn schon seit 1847 für wahnsinnig hielt. Während der Revolution war Kriege nach dem 2. Kongresse der Demokratischen Partei in Deutschland Mitglied von deren Zentral Komitee, um nach der Niederlage der Revolution in Berlin und Hamburg im Mai 1849 nach USA zurückzugehen. Er starb Ende 1850 oder Anfang $185 \mathrm{I}$ in einer Irrenanstalt in New York (freundl. Auskunft von Herrn Boris Nicolaevsky). ${ }^{5}$ Edmund J. James und Milo J. Loveless, A Bibliography of Newspapers published in Illinois prior to I 860 (Publications of the Illinois State Historical Library, Nr. I, Springfield I 899), S. 20.

6 Uber diesen Chicago und sein Deutschthum, S. I09, I I 2. Höffgen und Schneider zeichneten gemeinsam für Druck und Verlag, bis im September I 861 noch ein "Co." hinzukam; vom Oktober des Jahrs ab firmierten Schneider und L. Brentano, dessen Name von Mittc April 1862 an allein im Zeitungskopf erschien.

7 Brief an seinen Vater vom 30. Juni I861, übers. in Caspari, op. cit., S. 275. 
Vereinigten Staaten gab 1, wurde die Staats-Zeitung so zum führenden republikanischen Sprachrohr des Westens. Bei einem besonderen Anlass sammelten sich nacheinander tausende vor det Geschäftsstelle an, um die letzten Nachrichten zu erfahren. ${ }^{2}$ Der Erfolg erklärte sich teilweise durch die benutzte „herrliche deutsche Muttersprache” 3 wie durch die zur Verfügung stehenden „eilf (sic) Dampfpressen" 4 , dank deren die Druckerei in der Madison-Strasse ${ }^{5}$ sich als grösste Amerikas bezeichnete. Die Aufmachung der Zeitung übertraf auch die mancher Rivalen in englischer Sprache. Nachrichten, Leitartikel und Berıchte müssen jedoch ihre besondere Wirkung gehabt haben. $\mathrm{Zu}$ den Verfassern der letzteren gehörte mehrere Jahre lang ein Pariser Korrespondent. Es war Moses Hess.

Der bemerkenswerte Rheinländer, der Karl Marx auf den Sozialismus hinstiess, Friedrich Engels dafür gewann, eine ähnliche Pionierrolle im frühen Zionismus spielte und den Fachleuten zufolge auch die moderne Auffassung vom Atom vorwegnahm ${ }^{6}$, verdient demnach ebenfalls einen Platz in der Geschichte der amerikanischen Presse. Wer wöchentlich ${ }^{7}$ seine Berichte las, erhielt dadurch ziemlich ausführliche Übersichten aus der französischen Hauptstadt ${ }^{8}$, deren wert-

1 W.I.S.Z., Bd. XXIII (1869), Nr. I3, S. 4. Bessie Pierce benutzt einige von diesen noch unerschöpften Minen in ihrer materialreichen History of Chicago, Bd. II, New York I940, und veranlasste die Übersetzung von Auszïgen aus deutschsprachigen Organen (siehe The Chicago Foreign Language Press Survey, Chikago 1942). Der Verf. dankt der Newberry-Bibliothek dafür, ihre umfangreiche Sammlung einsehen zu dürfen.

2 T.I.S.Z., Bd. XXIII (1870), Nr. I92, S. 4.

3 W.I.S.Z., Bd. XXIII (1869), Nr. 35, S. 2.

${ }^{4}$ Ibid., Nr. 45 , S. 7.

${ }^{5}$ Nr. 104 zwischen Dearborn- und Clarkstrasse. Ein Bild des späteren Zeitungsgebäudes in Eugen Seeger, Chicago, die Geschichte einer Wunderstadt, Chikago 1892, S. 4 Ir. Eine wissenschaftliche Behandlung des Blatts unternimmt Andrew Jacke Townsend, The Germans of Chicago (Diss.) Chikago 1927, passim. Zahlreiche Hinweise auf die StaatsZeitung cnthält Carl Wittke, The German-Language Press in America, University of Kentucky Press, I957.

- Siehe Theodor Zlocisti, Moses Hess der Vorkämpfer des Sozialismus und Zionismus: 1812-1875, 2. rev. Ausg., Berlin 1921, namentlich S. 99.

${ }^{7}$ Manchmal erschienen zwei Wochenberichte in der gleichen Nummer, manchmal kam einer in der Tagesausgabe heraus. Wir halten uns für die Berichte durchgängig an die Wochenausgabe. Einige in der Tagesausgabe enthaltene Hess-Berichte wird Horace Weiss demnächst in einer Dissertation über Deutschen Sozialismus in den vierziger Jahren an der Columbia-Universität behandeln.

${ }^{8}$ Einige kamen ,vom Rhein", so ein vom 2. Juli datierter Bericht, W.I.S.Z., Bd. XXI ( 1867 ), Nr. 29, S. 5, in dem es inmitten cines guten Passus über Freiheit und Einheit seit I 848 heisst : „Der Krieg um der Machtvergrösserung willen wird hier, wie überall, verabscheut"; ein ebensolcher vom I 2. Juli (ibid., Nr. 30, S. 4-5) mit dem Schluss, „dass jeder Sturm nur die Luft reinigen und Entwicklung des modernen Lebens beschleunigen kann"; schliesslich einer vom I 2. Juni, Bd. XXII (1 868), Nr. 26, S. 4-5, dessen fesselnder Vergleich zwischen französischer und deutscher Kultur die Warnung enthält, dass Europas wirkliche Gefaht von Russland komme mit seinem „Bodencommunismus”. 
vollster Bestandteil, auf lange Sicht hin beurteilt, in der prophetischen Begabung des Korrespondenten gelegen haben dürfte.

Von jenen politischen, sozio-ökonomischen und technischen Vorhersagen seien an dieser Stelle zwei Beispiele gegeben. Zum Suezkanal, „dem grossen Unternehmen... im Geburtsland der jüdischen Nation, die man auch jetzt wieder an der Colonisation dieses Landstriches betheiligen möchte" 1 , bemerkte der sonst unentwegte Gegner seines Bauherrn mit beachtlicher Objektivität: „Das zweite Empire kann sich damit trösten, wenigstens Ein grosses nützliches Werk gefördert und zur Ausführung gebracht zu haben"2. Nachdem er die Vorgeschichte des Kanals gegeben und wiederholt hatte, dass das Projekt erst in den fünfziger Jahren „ernstlich in Angriff genommen und von der kaiserlichen Regierung in jeder Weise unterstützt" ${ }^{3}$ worden war, schloss Hess mit der Prophezeiung, „dass mit der Vollendung dieses Unternehmens, das so zu sagen mit dem Empire begonnen hat, auch dieses Letztere sein Ende erreicht zu haben scheint". ${ }^{4}$ Das wurde neun Monate vor dem Zusammenbruch Napoleons des Dritten festgestellt.

Skeptisch gegenüber dem ihm bekannten deutschen Konstrukteur eines Flugapparats, der von dem französischen Kaiser gefördert wurde ${ }^{5}$, sagte Hess nichtsdestoweniger - im Jahr 1868 ! - mit ähnlicher Treffsicherheit voraus, der mechanische Vogel werde, „wenn er gelingt, den Hinterladern, sowie den grossen und kleinen Kanonen, eine tötliche Concurrenz machen, da er im eigentlichen Sinne des Wortes eine 'fliegende' Artillerie werden kann". ${ }^{6}$ Der Prophet konsultierte Erfinder wie Antoine A. Chassepot ${ }^{7}$ und Fachleute wie Kolonel Rüstow ${ }^{8}$, trat also weder als religiöser Schwärmer, noch als Dichter oder Fantasieschriftsteller auf. Deshalb sollten derartige Prophezeiungen wohl nicht als einfaches Herumraten beiseitegeschoben werden.

Dass Hess der Verfasser der Pariser Berichte war, und wann er seine Mitarbeit begann und beendete, kann aus äusseren und inneren Kriterien festgestellt werden. Einmal haben sich Briefe der Redaktion

1 Ibid., Bd. XX(1866), Nr. I9, S. 5.

2 Ibid., Bd. XXIII (1869), Nr. 50, S. 7.

3 Ibid.

4 Ibid.

5 Ibid., Nt. 5 r, S. 3.

- Ibid., Bd. XXII (1868), Nr. 23, S. 5.

7 Siehe ibid., Bd. XXI (1867), Nr. 8, S. 3 .

${ }^{8}$ Siehe ibid., Bd. XXII (1868), Nr. 24, S. 5. 
an ihn erhalten, von denen weiter unten noch die Rede sein wird. ${ }^{1}$ Andererseits bezog die "Staats-Zeitung" sich in einer Züricher Korrespondenz vom 20. Dezember (1864) auf die alten „Kämpen Fritz Engels, Moses Hess, Karl Marx und Georg Herwegh" 2 als Mitarbeiter des soeben ins Leben tretenden "Sozial-Demokrat”. In einem ,am Vorabend des Osterfestes '65" datierten Artikel brachte die „Staats-Zeitung" hiernach ,von unserem in Paris befindlichen schweizerischen Correspondenten" die den im Allgemeinen Deutschen Arbeiterverein ausbrechenden Konflikt betreffende Bemerkung: „Ich bin überzeugt, M. Hess, der alte getreue Socialist und Vater der alten Partei der Socialisten in Deutschland, behandelt die Sache, wie er es als Pariser Correspondent für den Social-Demokraten thut, ganz richtig. Er kennt die Schwachheiten der Londoner Fraktion (Marx und Engels) so durch und durch und entpuppt das Streben dieser ehrgeizigen Leute so ruhig und klar...".3 Zu dieser Empfehlung eines Mannes, dessen Namen solcherart einst vor dem Marxens genannt wurde, meinten die Herausgeber, ohne sich in den Streit selbst einmischen zu wollen, „dass die Herren Marx und Engels nur ehrenhaft und prinzipientreu handelten, wenn sie auf's nachdrücklichste gegen jede Annäherung des Arbeiterbundes an die reaktionäre und perfide innere Politik des Hrn. v. Bismarck remonstrirten". ${ }^{4}$ Beide Erwähnungen Hessens machen es unwahrscheinlich, dass er zu jenem Zeitpunkt bereits in Chikago mitarbeitete.

Am I6. August des gleichen Jahrs stellte eine vom 2r. Juli datierte Abhandlung "von der unaufhaltsamen demokratischen, oder sagen wir geradezu republikanischen Bewegung der westeuropäischen Völker" ${ }^{5}$ sich indirekt, durch die Numerierung eines nachfolgenden Berichts ${ }^{6}$, als Anfang einer Serie von 96 weiteren vor. Dieser folgte eine zweite Serie von 96 Artikeln und danach eine dritte, welche fünf Jahre später mit Nr. $25^{7}$ Hessens Mitwirkung an der Staats-Zeitung beendet zu haben scheint. Als Pariser Korrespondenz gekennzeichnet - teilweise sicher schon deshalb, weil der Zeitung vorgeworfen

1 Prof. Edmund Silberner, der in der ersten Ausgabe seiner bekannten Hess-Bibliographie (Moses Hess: an annotated biliography, Burt Franklin Bibliographical Series Nr. 4, New York, 1951, S. vi, cf. S. 62) die Frage der Autorschaft noch offen liess, hatte die Freundlichkeit, in einem Schreiben vom 30. Juni 1952 den Verf. daran zu erinnern, dass er selbst bei der Sichtung des Hess-Nachlasses in den dreissiger Jahren die Staats-ZeitungsBriefe bemerkt hatte. (Siehe Helmut Hirsch, Einiges über Moses Hess, Vater der deutschen Sozialdemokratie, in: Denker und Kämpfer, S. 83-110).

2 W.I.S.Z., Bd. XIX (1865), Nr. 3, S. I.

3 Ibid., Nr. 20, S. I.

4 Ibid.

5 Ibid., Nr. 33, S. I.

" „V. Paris, 18. August," ibid., Nr. 37, S. I.

7 Ibid., Bd. XXIV (1870), Nr. 26, S. 1-2. 
wurde, sie besitze keine Originalberichte von auswärts ${ }^{1}$ - waren diese Überblicke niemals unterzeichnet, was als Vorsichtsmassnahme gedacht war. ${ }^{2}$ Ihnen ging jedesmal eine möglicherweise von der Redaktion hergestellte Inhaltsübersicht voraus; gelegentlich versah erstere auch einen Beitrag mit markierten Einschaltungen oder Fussnoten. Im Rampenlicht des Wochenreferats stand gewöhnlich Frankreich. Darüber hinaus wurden der europäischen Politik, der Börsenlage und anderen Fragen von öffentlichem Interesse Beachtung geschenkt. Obschon der Pariser Beobachter, offenbar wiederum aus Sicherheitsgründen, in der Regel wenig von sich selbst erzählte ${ }^{3}$, verheimlichte er nicht, dass ihn das breite Volk am meisten interessierte. Als ganz spezieller Freund enthüllte er sich gegenüber der organisierten Arbeiterschaft.

Bereits in seiner ersten Einsendung wies Hess auf die junge Internationale Arbeiter-Association hin. „Die Idee dazu”, erinnerte er sich im Gegensatz zu manchen anderen ", „,ist ursprünglich vor einigen Jahren von Garibaldi ausgegangen". ${ }^{5}$ Im zweiten Beitrag stellte der neue Mitarbeiter die gleichzeitige Reorganisierung der sozialistischen Bewegung in Deutschland dar. „Niemand kann im Voraus sagen”, war seine Weissagung hierzu, „wie weit diese Volksdemonstrationen führen werden”. ${ }^{6}$ Der dritte Artikel gipfelte in der Behauptung: „Die einzige Hoffnung für Deutschland liegt doch nur in den deutschen Arbeitern, die täglich mehr zu politischer Reife gelangen". ${ }^{7}$ Auf den dahingeschiedenen Ferdinand Lassalle bezugnehmend, "der sie vereint hat und zum Aufstand hätte führen können" 8, erkannte der Korrespondent dennoch, dass die Arbeiter nur gemeinsam mit dem Bürgertum vorgehen durften. „Es gibt freilich in Deutschland noch einige Enthusiasten, die eine Erhebung der Proletarier-Partei erwar-

${ }^{1}$ Siehe die ziemlich grobe Notiz gegen die „Westliche Post”, wobei Hess ungenannt als einer der ausgezeichneten Schriftsteller erscheint, die als Korrespondenten beschäftigt würden (ibid., Bd. XIX - die Bandnummer ist verdruckt - 1865, Nr. 45, S. I).

${ }^{2}$ Siehe ibid., Nr. 45 , S. 2.

${ }^{3}$ Ausnahmen werden weiter unten besprochen. Dass er sogar der Redaktion gegenüber seine Adresse in den Briefen fortliess, ging dieser nicht ein. Nachdem sie die Anschrift verlegte hatte und der Korrespondent über einen Monat sein Geld nicht erhielt und mahnte, schrieb Hermann Raster in einem Brief an Hess vom I8. Januar 1870: „eine Mahnung mit Hinzufügung der Adresse würde viel schneller zum Ziele geführt haben, als solche Mahnungen obne solche Hinzufügung". Für die Zurverfügungstellung von sechs Mitteilungen an Hess ist der Verf. dem Internationalen Institut für Sozialgeschichte sehr verbunden.

${ }^{4}$ Cf. Helmut Hirsch, Aufstieg und Niedergang der Ersten Internationale, in: Denker und

Kämpfer, S. 1 29-I 48 .

5 W.I.S.Z., Bd. XIX (1865), Nr. 33, S. I.

${ }^{6}$ Ibid., Nr. 34, S. I.

7 Ibid., Nr. 35 , S. r.

${ }^{8}$ Ibid., Bd. XX (1866), Nr. 19, S. 3. 
ten, wenn man dem Vaterlande das Messer an die Kehle setzen wird", meinte er. „Es hat sich aber in der modernen Geschichte noch nie gezeigt, dass die Proletarier, wenn sie nicht mit der Bourgeoisie vereint handeln, es zu etwas Erspriesslichem bringen können". ${ }^{1}$ Als nächstes schilderte er eingehend die Schikanierung des Organs der sozialistisch gesinnten französischen Arbeiterkooperativen und erwähnte den Zusammentritt der Internationale in Genf. „Die Fragen, welche dort diskutirt werden, sind von höchstem Interesse und die Diskussion scheint einen ernsten wissenschaftlichen Charakter anzunehmen". ${ }^{2}$ Daran schlossen sich nach einer Weile Nachrichten über die Verhaftung einiger Pariser Mitglieder der Internationale und die bevorstehende Bildung der "Sozial-demokratischen Partei" 3 in Deutschland, deren Programm den Chikagoer Lesern ebenfalls vorgelegt wurde. ${ }^{4}$

Obschon selbst Deutscher und für Deutschamerikaner schreibend, stand Hess den deutschen Sozialisten nicht unkritisch gegenüber. Marx, sagte er einmal, hätte sich von bonapartistischen Arbeiterdelegierten düpieren lassen. ${ }^{5}$ Im Gegensatz zu seiner vormaligen Betonung der Reife und Rolle des deutschen Proletariats behauptete der Berichterstatter jetzt auch: „Die grosse Masse des Volkes ist trotz der forcirten Agitation der Lassalianer (sic) in Deutschland noch weit weniger reif zu einer selbständigen Vertretung ihrer Interessen, als in England und Frankreich". ${ }^{6}$ Nur etwa einen Monat später hiess es indessen: „Die internationale Arbeiter-Association ist seit der kurzen Zeit ihres Bestehens schon eine Macht geworden, welche mit den Kapitalisten auf gleichem Fusse unterhandelt, und welche vielleicht bald Gesetze diktiren kann" ", wurde sie doch „mit grosser Umsicht von Londen aus dirigiert". ${ }^{8}$

Als in den Wohnungen der französischen Mitglieder der Internationale Haussuchungen vorgenommen wurden, brachte Hess diese Verfolgung zur Kenntnis der amerikanischen Leserschaft zusammen mit einem das bonapartistische Regime gleichfalls kompromittierenden Theaterskandal und - der grössten Kälte (minus 12 Grad Celsius), die er seit zwanzig Jahren in Paris erlebt hätte. ${ }^{9}$ Letztere Angabe musste

1 Ibid. Sperrung im Original.

2 Ibid., Nr. 39, S. 3.

3 Ibid., Nr. 49, S. 5 .

4 Ibid., Bd. XXI (1867), Nr. 4, S.3.

5 Ibid., Nr. 5, S. 3.

6 Ibid., Nr. 9, S. 5 .

'Ibid., Nr. 1 5, S. 2 ; siehe auch S. 5.

${ }^{8}$ Ibid., Nr. 38 , S. 4-5.

9 Ibid., Bd. XXII (1868), Nr. 3, S. 4-5. 
den stets geschäftigen Agenten der Pariser Polizei ${ }^{1}$, falls sie die Korrespondenz zu sehen bekamen, noch nützlicher sein als eine bereits zu Anfang gemachte Anspielung auf das Altern des Schreibers. ${ }^{2}$ Er hatte seine Herkunft verraten, als er im Zusammenhang mit deutscher Ängstlichkeit und Uneinigkeit von ,den jämmerlichen Zuständen unseres Vaterlandes" 3 sprach. Aus einer Mitteilung über die Choleraepidemie erfuhr man, dass er in der Nähe des Pariser Stadtviertels der Battignolles wohnte ${ }^{4}$, wo es eine Sektion der Internationale gab. ${ }^{5}$ Er war sogar so unvorsichtig gewesen, sich als kritischer Besucher eines religionswissenschaftlichen Vortrags an der Sorbonne zu erkennen zu geben, der ,aus Neugierde" 6 teilgenommen und seine Augen immer auf die Statue Voltaires gerichtet habe - welche Geste allerdings wohl mehr bildlich als wörtlich zu verstehen war. Noch weiter lüftete der Berichtende sein Incognito in Verbindung mit den Samstagabendveranstaltungen in den Räumen des deutschen Turnvereins in Paris. Hier trafen sich junge Leute aus allen Schichten zur Diskussion „bis gegen Mitternacht". ${ }^{7}$ Er sei gebeten worden, über „das Verhältnis von Kapital und Arbeit" ${ }^{8}$ einen Vortrag zu halten, erklärte der Schreiber, was keinen schlechten Anhaltspunkt für seine Identität bot. Gelegentlich von Arbeiterunruhen in Basel zitierte er schliesslich einen, wie er mitteilte, ihm vom Zentralkomitee der Internationale zugestellten Aufruf. ${ }^{9}$ Aufschlussreicher konnte ein Umstand kaum sein.

War es Kamouflierung Paris und Chikago ${ }^{10}$ gegenüber, wenn Hess kurz darauf Kommunisten und Proudhonisten als „Utopisten" 11 betitelte? Gleich zu Beginn seiner Tätigkeit distanzierte er sich indirekt vom utopischen Sozialismus der Vergangenheit und revolutionären Sozialismus der Gegenwart.12 Im Anschluss an zwei Berichte über die Pressepläne der französischen Sozialisten und die Einstellung der deutschen, unter denen der autoritäre Lassalleanismus sich über-

1 Cf. Helmut Hirsch, Marx in den Augen der Pariser Polizei, in: Denker und Kämpfer, S. I23-1 28.

${ }^{2}$ W.I.S.Z., Bd. XIX (1865), Nr. 48, S. 2.

3 Ibid., Nr. 37 , S. I.

4 Ibid., Nr. 45 , S. 2.

${ }^{5}$ Siehe die meisterhafte Arbeit von Charles Rihs, La Commune de Paris: sa structure et ses doctrines; Études d'Histoire Économique, Politique, et Sociale, Bd. XII, Genf r955, S. 53 .

6 W.I.S.Z., Bd. XX (1866), Nr. Is, S. I.

7 Ibid., Bd. XXII (I 868), Nr. 5 r, S. 2. (Seitenzahlen falsch numeriert)..

${ }^{8}$ Ibid. Siehe auch ibid., Bd. XXIII (1 869), Nr. 22, S. 5.

9 Ibid., Nr. 3, S. 2. Der Bericht enthält auch einen antiklerikalen Angriff.

10 Zur Haltung der Staats-Zeitung cf. ibid., Nr. 16, S. 2.

11 Ibid., Nr. I5, S. 3.

12 Ibid., Bd. XIX (I865), Nr. 44, S. x. 
lebt habe ${ }^{1}$, sprach er ferner in einer Betrachtung über die mehr praktische und politische Natur der sozialen Frage seit den vierziger Jahren von Londoner „Agitatoren”. ${ }^{2}$ Sie hätten bei den in Belgien und der Schweiz ausgebrochenen Arbeitskonflikten ,ihre Hand im Spiel". 3 Ohne spezielle Sympathie für die Linke gab sich auch die auf die Gerichtsverhandlungen gestützte Darstellung eines Pariser Prozesses gegen die Internationale. ${ }^{4}$ Doch verschwieg der Korrespondent nicht, dass er dem Brüsseler Kongress der Internationale (vom 7. September I $868 \mathrm{ab}$ ) ,als Augenzeuge” 5 beiwohnte. „Von einer mehrwöchentlichen Reise durch Belgien und Deutschland zurückgekehrt" 6, konnte er damit eine beachtenswerte Skizze ihres Sekretärs liefern, der gemeinhin als blosses Instrument von Marx angesehen worden ist." Die bald engagierte, bald degagierte Art der Berichterstattung verstärkt den Eindruck einer gewissen Duplizität, den unser Held auch sonst zuweilen machte. ${ }^{8}$

Hessens Intimität mit der äussersten Linken mag auch die nichtsozialistischen Leser im Mittleren Westen befremdet haben. Es musste sie interessieren, wenn er ihnen beschrieb, wie er auf dem Montparnasse-Boulevard stand, als das kaiserliche Paar vorbeikam und nur von wenigen Passanten begrüsst wurde. ${ }^{9}$ Doch wollten sie etwas von Hegels „Maulwurf der Weltgeschichte"10 wissen? Es klang zum mindesten apologetisch (und setzte demnach ein Befremden voraus), wenn der sozialistenfreundliche Theoretiker nach einem kleinen geschichtsphilosophischen Exkurs hinzusetzte: „Sicher haben es viele Ihrer Leser bezweifelt, wenn ich es schon längst und wiederholt hervorhob, dass das 'rothe Gespenst' dem heutigen Frankreich keine Furcht mehr einflösst. Man kann den auswärtigen Lesern deshalb keinen Vorwurf machen; die innern Zustände Frankreichs werden selbst von den hier lebenden Ausländern verkannt und falsch beurtheilt. Man muss nicht nur sehr lange unter diesem Volke, sondern auch mit ihm gelebt haben, um es gehörig würdigen zu können". ${ }^{11} \mathrm{Je}$ weniger man sich aber vor dem "rothen Gespenst" fürchte - sagte

1 Ibid., Nr. I4, S. 5 und Nr. Is, S. 4.

2 Ibid., Nr. I6, S. 5.

3 Ibid.

4 Ibid., Nr. 20, S. 5.

5 Ibid., Nr. 37, S. 5.

- Ibid., Nr. 40, S. 5 .

7 Wiedergegeben in Helmut Hirsch, Aufstieg und Niedergang der Ersten Internationale, in: Denker und Kämpfer, S. I 40-141.

${ }^{8}$ Siehe Einiges über Moses Hess, ibid., S. 91-96.

W.I.S.Z., Bd. XXIII (1869), Nr. 24, S. 4-5.

10 Ibid., S. 4.

11 Ibid. Im Original gesperrt. 
der Prophet in einem besonders apokalyptisch gestimmten Bericht die Pariser Kommune von I 871 voraus - „desto mehr wird in Wirklichkeit die nächste französische Republik eine 'rothe', d.h. eine solche sein, welche den Forderungen der arbeitenden Klassen Rechnung tragen, und das Prinzip der Gleichheit neben dem der Freiheit verwirklichen wird." 1

In einer Bemerkung über die Armut der Internationale, die kaum einen Generalsekretär besolden könne, bewies Hess erneut, dass er das Funktionieren der Organisation von innen heraus kannte. ${ }^{2}$ Angehörige der Klasse, die ihm am Herzen lag, waren es, welche er in der Art eines modernen Reporters interviewte, während eine neugewählte französische Kammer ihre erste Sitzung abhielt. Konkordienplatz und Tuileriengarten, die den Platz mit dem Palais der gesetzgebenden Versammlung verbindende Brücke, sämtliche Quais diesseits und jenseits des Platzes, kurz, die nahe und ferne Umgebung der Deputiertenkammer - so schilderte die Reportage anschaulich waren mit vielen tausenden von Menschen aus allen Klassen angefüllt. Sie begrüssten die Abgeordneten der Opposition bei ihrer Ankunft und beim Verlassen des Parlaments mit lauten Vivats. Von Mittag bis sechs Uhr blieb die Masse, durch ein grosses Aufgebot an Militär überwacht, an diesen Orten stationiert, ohne dass es zum geringsten Zusammenstoss kam. Man vertrieb sich die Zeit mit ernsten und ruhigen Gesprächen über die politische und sozialpolitische Lage. „Es war weder blosse Neugier, noch auch lediglich der Wunsch, die neuen Oppositions-Deputirten zu begrüssen, welches den bessern Theil des Volkes herbeizog", ging die sozialgeschichtlich fesselnde Schilderung weiter. "'Wir wollen der Regierung zeigen', sagten mir Arbeiter, die ich wegen der Ursache ihres Hierseins befragte, 'dass wir hinter unsern Abgeordneten stehen und sie nöthigen Falls gegen Gewaltstreiche zu schützen entschlossen sind" ". ${ }^{3}$ Es sei wunderbar, bis wohin der politische Sinn heute gedrungen sei, lautete ein Zusatz. Knaben von zehn oder zwölf Jahren wüssten oder fühlten instinktiv, um was es sich handele. Zu solchen an einen der Klassiker der Revolutionsgeschichte gemahnenden Beobachtungen gehörte gewiss mehr als journalistische Technik.

Ein ähnliches Urteil drängt sich einem auf, wenn man die nicht eben unparteiische ${ }^{4}$ Beschreibung des Eisenacher Kongresses liest, mit

1 Ibid., Nr. 43, S. 5. $\quad 2$ Ibid., Nr. 27, S. 3. $\quad 3$ Ibid. Nr. 29, S. 5.

4 Dass Hess "die erste marxistische Partei auf deutschem Boden"(Georg Eckert in seiner wertvollen dokumentarischen Studie: Aus den Anfängen der Braunschweiger Arbeiterbewegung, Braunschweig, I955, S. 111, recht einseitig unterstützte, lag z.T. an der Propaganda, der er selbst ausgesetzt war (siehe W. Brackes Brief an ihn vom 27. Juni 1869, ibid., S. 22). Hessens Bericht war - vielleicht durch die andere Einstellung eines Setzers? so mit Druckfehlern durchschossen, dass wir diese hier der Verständlichkeit halber verbessert haben. 
welcher der ehemalige Kritiker der unreifen europäischen und namentlich deutschen Arbeiter die Gründung der sozialdemokratischen Arbeiterpartei feierte. „Welch' ein herrliches Schauspiel geht in diesem Augenblicke in Deutschland vor sich!” rief Hess begeistert aus. „Die radikalsten sozial-demokratischen Arbeiter haben in Eisennach einen Congress abgehalten, um sich von dem elenden Volksverführer loszumachen, der sie im Namen des Messias Lassalle und im Interesse der preussischen Feudalen gegen die radikale und republikanische Bourgeoisie hetzen wollte". "Weit mehr als hunderttausend Arbeiter aus Nord- und Süddeutschland, aus Österreich und der deutschen Schweiz seien dort vertreten gewesen, hiess es weiter. Mit den von der Gräfin Hatzfeldt besoldeten Jungen, etwa hundert an der Zahl, die mit falschen Mandaten angekommen seien, um den Kongress zu stören, sei man bald fertig geworden. Dann habe sich die neue sozialdemokratische Volkspartei konstituiert, deren Organ, „der Volksstaat", in Leipzig erscheinen werde. Sie stehe einerseits mit der süddeutschen Volkspartei und der radikalen norddeutschen Fraktion Johann Jacobys, andererseits mit der Internationalen ArbeiterAssoziation, deren Program sie im wesentlichen angenommen habe, in Verbindung. „Man ersieht daraus, dass die deutschen Arbeiter überall zu Verstande gekommen sind und vor allen Dingen die politische Freiheit erkämpfen wollen, dass sie allen diktatorischen Gelüsten, wenn auch angeblich zu sozialistischen Zwecken, für immer den Rücken gekehrt haben, und dass die nächste europäische Revolution sie ebenso reif zum praktischen Handeln finden wird, wie die Arbeiter Frankreich's, England's und der übrigen europäischen Culturstaaten". 2

Anlässlich des Baseler Kongresses der Internationale, dem er wiederum beiwohnte, machte Hess sich um die Geschichte der Chikagoer Arbeiterbewegung verdient. Er beschrieb zunächst das unerwartete Erscheinen des von hier entsandten, soeben (am 9. September I 869) aus Philadelphia angekommenen Abgeordneten der National Labor Union, Andrew C. Cameron. Er wurde mit allgemeiner Begeisterung und einer besonderen Begrüssung durch den Vorsitzenden empfangen. Mehr hörte der Leser über die zwei Tage später gehaltene Rede des Amerikaners vor dem Kongress. Er lehnte Republikaner und Demokraten gleichermassen ab. „Die Interessen der Arbeiter könnten nur von Arbeitern vertreten werden, sowohl in den Staaten, wie in der Union”, referierte der Korrespondent. „Die internationale Arbeiterassociation sei berufen, die Arbeiter aller Länder zu befreien,

1 W.I.S.Z., Bd. XXIII (1869), Nr. 36, S. 4.

2 Ibid. 
und die amerikanischen Arbeiter wollten durch ihn ihre Sympathien diesem Bunde bezeugen. Unter dem Banner 'Wahrheit, Gerechtigkeit und Gleichheit' würden sie gemeinsam mit der Internationalen einem sicheren Siege entgegen gehen". ${ }^{1}$ Die in diesem Fall nicht ganz irrige Siegesgewissheit der damaligen Sozialisten spiegelte sich, wie ebenfalls mitgeteilt wurde, in dem von der letzten Sitzung des Kongresses einstimmig gefassten und stürmisch applaudierten Beschluss wieder, im nächsten Jahr in Paris zusammenzukommen. So sicher war man des Sturzes der kaiserlichen Regierung, unter deren Herrschaft an einen Pariser Kongress der Internationale nicht zu denken war.

„Die Schauspieler können wechseln; das Schauspiel bleibt überall dasselbe bis zum Schlusse" 2, hatte Hess schon zu Anfang des Jahrs ausgerufen. Er dachte dabei an den französischen Staat. „Dieser Schluss kann nur sein das Ende der achtzehnjährigen Comödie, die nach dem Untergang der französischen Republik von I 848 durch einen Napoleon aufgeführt worden, und die sowohl in nationaler wie internationaler Beziehung täglich den Völker- und Volksfrieden bedroht". ${ }^{3}$ Eschatologisch war dieses Denken aber nicht nur gegenüber Regierungs- und Gesellschaftsform, sondern auch gegenüber der römischen Kirche. Die Katholiken aller zivilisierten Länder, Deutschland, Frankreich, Italien, Spanien, Nordamerika, „, in welchen Ländern der katholische Clerus zum Theil schon gegen die römischen Tendenzen protestiert hat” 4, würden sich von Rom lossagen. „Dann kann der Papst nach Jerusalem gehen und sich dort mit dem Papstthum begraben lassen". 5

Mit wöchentlich wachsender Zuversicht versprach Hess namentlich das herannahende Ende des kaiserlichen Regimes. Das bedeutete einen völligen Umsturz, denn "die Zeiten der Emeuten sind vorüber; es kann keine andern mehr geben, als die von der Polizei organisierten". ${ }^{6}$ Schon streikten - von Engländern und Deutschen unterstützt, wie der Pariser Freund der Internationale nicht zu sagen vergass - I0.000 "Ladendiener" 7 in der Hauptstadt. Die Streikenden bildeten ihre

1 Ibid., Nr. 41, S. 2. Wir bringen nur einen Auszug des von Hess Berichteten. Dass er als Berliner Delegierter im Vorstand des Kongresses sass und mit Cameron stimmte (siehe James Guillaume, L'Internationale: Documents et Souveniers, Paris 1905, II. T. S. 204, 223) wurde natürlich nicht verraten.

2 W.I.S.Z., Bd. XXIII (1869), Nr. 2, S. 2.

3 Ibid.

4 Ibid., Nr. 42, S. 2.

5 Ibid. Die Lebensdauer der römischen Kirche so zu unterschätzen, war junghegelianisches Gedankengut (siehe Helmut Hirsch, Karl Friedrich Köppen, der intimste Berliner Freund Marxens, in: Denker und Kämpfer, S. $7^{6}$ ).

- Ibid., Nr. 45, S. 5 .

7 Ibid. 
eigenen, von anderen Arbeitnehmern unterstützten Konsumgenossenschaften. ${ }^{1}$ Politisch bedeutungsvolle Gerichtsverhandlungen konnten damit rechnen, „eine grosse Volksmasse” ${ }^{2}$ herbeizuziehen. Kurz, „das Empire ist moralisch vernichtet; ein Windstoss kann das morsche Gebäude in eine materielle Ruine umwandeln". ${ }^{3}$ Wie aber das liberaler gewordene Kaiserreich „,von Station zu Station" 4 über sich selbst hinaus zu seinem Geg enteil, zur Republik, getrieben wurde, so wurden die gemässigten Republikaner unwillkürlich in den Radikalismus, die radikalen in den Sozialismus hineingedrängt; der stets Theoretisierendezeigte dieses an einigen geschickt gewählten Beispielen.

Ein weniger radikal ausgerichteter Oppositionsführer wie Alexander August Ledru-Rollin wurde mit dem Ansteigen des revolutionären Fiebers bei Hess mit einemmal aus einem Bundesgenossen zum halben Gegner. „Als ächter Vertreter der radikalen Bürgerklasse, die sich vor ihrem eigenen Schatten, vor den revolutionären Arbeitern fürchtet", höhnte er, ohne an seine eigene vormalige Klassenbündnispolitik zu erinnern, ,legte er seine Löwenhaut rechtzeitig ab und zeigte sich als das, was er ist, als der Leithammel einer geduldigen Schafsherde, die in dem Volke, welches ihre Kämpfe durchgeführt hat, wohl noch den Schäferhund liebt, der sie gegen den Wolfsappetit einer alles verschlingenden Regierungsgewalt schützt, aber nicht den Jagdhund, mit dessen Hülfe sie ihren Feind angreifen und vertilgen könnte. Das Volk ist ihr zu intelligent geworden, und sie fürchtet, dass es sich emancipiren und auch ein wenig für eigene Rechnung jagen würde". ${ }^{5}$ Die Proletarier würden sich in der Tat selbst befreien und ,je länger der Sturz des Empire verzögert wird (endete einer der häufigen Kommentare über die Dialektik der Revolution) einen desto röthern Charakter nimmt die Revolution an, unter deren Schlägen es und mit ihm das ganze monarchische System in Europa zusammenbrechen wird". ${ }^{6}$ Wie naturwissenschaftlich das hier entwickelte Geschichtsbild konzipiert war, lässt sich aus einer bei anderer Gelegenheit gemachten Bemerkung erkennen. Es hänge weder von der Presse, noch von den Persönlichkeiten der Opposition mehr ab (hiess es dort), ob eine Bewegung aufzuhalten sei, die, einmal begonnen, ihren eigenen Gesetzen folge. Zwar wisse man nicht, ob die Revolution an diesem oder jenem Tag ausbrechen werde. Gerade französische Massen aber ähnelten einer Naturkraft. „Hier hört, wie in der Natur, jede Refle-

1 Ibid., Nr. 47, S. 3.

2 Ibid., Nr. 45, S. 5 .

3 Ibid., Nr. 47, S. 3.

4 Ibid., Nr. 52, S. 4.

5 Ibid., Nr. 50, S. 7.

- Ibid., Nr. 52, S. 4. 
xion, jede bedächtige Überlegung, jede Willkür auf; ein guter Logiker kann nach Verlauf einer gewissen Beobachtungszeit ebenso sicher eine grosse französiche Volksbewegung vorherberechnen, wie ein guter Mathematiker nach einigen astronomischen Beobachtungen die Bahn eines Kometen". ${ }^{1}$

I I.

Will man aus zweihundert langatmigen Dokumenten auf zwanzig Seiten die den Historiker interessierende Substanz herausziehen (wie es unsere Absicht ist), dann darf nicht vergessen werden, dass der Massstab der Auslese eine kaum zu vermeidende Einseitigkeit bedingt. Wir haben dem Sozialpolitiker Hess besondere Aufmerksamkeit geschenkt, einmal weil er dafür ein Fachmann mit seltenen Kenntnissen war, und zum zweiten weil seine Bedeutung für die Sozialgeschichte im allgemeinen nicht genügend berücksichtigt worden ist. Übersehen wir indessen nicht, dass unser Material auch nach anderen Gesichtspunkten hin geordnet werden kann. Als Reporter zeichnete der Korrespondent der Staats-Zeitung sich beispielsweise am meisten durch einen Vergleich zwischen dem Karneval seiner Heimatstadt Köln und amtlichen Umzügen in Paris aus. ${ }^{2}$ Die biographisch interessanteste Einzelheit war seine Erinnerung an Heinrich Heines Urteil über den französischen Klerus. ${ }^{3}$ Die originellsten Gedanken der Artikelserien bergen drei Berichte. Der erste von diesen suchte eine Verbindung von Freiheit und Frieden. ${ }^{4}$ Der zweite pries die Liberalisierung der französischen Gesetzgebung über die Genossenschaften als „definitive Lösung" 5 der sozialen Frage. Der dritte erklärte das dialektische Verhältnis zwischen Otto v. Bismarck - „dem schlauen Grafen..., der freilich ein grosser Waghals ist" ${ }^{6}$ - und der französischen Reaktion.? Offenbar gibt es noch manche Ordnungsprinzipien für den Stoff. Um die von uns eingenommene Perspektive einigermassen auszugleichen, geben wir nachstehend einen aus dem Bereich der grossen Politik gewählten Auszug.

Hess malte hier ein für die Grundhaltung des neunzehnten Jahrhunderts typisches Bild vom mehr oder weniger automatisch zu erwartenden Fortschritt, wenn er schrieb:

1 Ibid., Nr. 44, S. 4.

2 Ibid., Nr. 52, S. 3.

${ }^{3}$ Ibid., Bd. XXIV (1870), Nr. I 3, S. 2. Über Mathilde Heine siehe T.I.S.Z., Bd. (1870),

Nr. 228, S. 2.

4 Der nachstehende Auszug.

5 W.I.S.Z., Bd. XXI (1867), Nr. 27, S. 5 .

B Ibid., Bd. XXIII (1869), Nr. 7, S. 2.

7 Ibid., Bd. XXI (1867), Nr. 29, S. 5 . 
„Man darf übrigens nicht glauben, dass Preussen, d.h. Bismarck, an dem unvermeidlichen Conflikte zwischen Frankreich und Deutschland unschuldig sei, wenn er auch im Grunde nur that, was er unter den gegebenen Umständen nicht lassen konnte. Es steht jetzt fest und wird von authentischster Seite bestätigt, dass Bismarck in Biarritz dem Kaiser Napoleon im eigentlichen Sinne des Wortes Holland und Brabant versprochen hatte, wenn sich Frankreich mit Preussen gegen Österreich verbinden wolle. Zu einem förmlichen Vertrage ist es allerdings nicht gekommen, aber daran ist der preussische Minister nicht schuld gewesen. Napoleon versprach ihm, wenn während des Krieges unglückliche Chancen bei Preussen eintreten sollten, zu des Letzteren Gunsten einzuschreiten. Nachdem es anders kam, als man erwartete, trug Preussen dem jedenfalls freundlichen Verhalten Frankreichs nicht die geringste Rechnung. Nicht hieraus freilich ist der preussischen Politik ein Vorwurf zu machen, wohl aber war es unverantwortlich von Seiten Bismarck's, gewissenlos an Frankreich fremde und deutsche Provinzen opfern $z u$ wollen, um dessen Freundschaft zu erkaufen. Aber auf dem Wege, den Preussen zur Machtvergrösserung einschlug, konnten nur solche Mittel angewendet werden, die allem Ehr- und Freiheitsgefühl Hohn sprechen.

Man behauptet zwar jetzt, dass Frankreich auch dann gegen jede Einigung und Stärkung Deutschlands wäre und stets gewesen sei, wenn diese Entwicklung auf dem Wege der Freiheit und Revolution vor sich gegangen wäre. Das kaiserliche Frankreich, allerdings! Aber es ist entschieden in Abrede zu stellen, dass das republikanische oder auch nur constitutionelle Frankreich sich jemals einer Einigung Deutschlands auf populärem Wege widersetzt hat und widersetzen würde. Welches Interesse könnten auch freie Völker daran haben, sich gegenseitig wegen eines Fetzen Landes zu bekämpfen oder auch nur eifersüchtig auf die Entwicklung ihres Nachbars zu sein, da nur Militärmächte sich aus Ehrgeiz und Herrschsucht bedrohen können, während freie Völker sich durch ihre Entwicklung nur gegenseitig fördern können. Arbeit, Handel, Industrie, Kunst und Wissenschaft heben sich in einem Lande nur um so mehr, je mehr sie auch im Nachbarlande gepflegt werden.

Aber freilich, so lange in den civilisirten Ländern noch rechtlose Zustände herrschen, aus welchen gewisse Klassen auf Kosten der anderen Nutzen ziehen - wie dies auch in Amerika der Fall war, so lange Sklavenstaaten existirten - sind Kriege unvermeidlich um so unvermeidlicher, wenn die Rechtlosigkeit in zwei grossen, 
auf ihre Macht stolzen Staaten in gleichem Grade besteht, und wenn diese Staaten Nachbarländer sind. Für Frankreich und Deutschland ist kein Heil zu erwarten, bis diese beiden Länder, und mit ihnen das übrige civilisirte Europa, sich von ihrer Militärherrlichkeit befreit haben.

Es ist die schönste Aussicht vorhanden, dass dieses in Folge des bevorstehenden Weltkrieges geschehen wird. Nachdem die hinterladigen Gewehre, gezogenen Kanonen und nagelneuen Handkanonen ihr Zerstörungswerk verrichtet haben, werden endlich den Völkern die Augen aufgehen, werden sie endlich einsehen, dass es besser ist, die grossen Fortschritte der Wissenschaft und Industrie zum Leben, als zum Ermorden der Menschen anzuwenden. In so fern ist es vielleicht schliesslich zum Nutzen der Völker, dass man den grossen Krieg nicht eher anfängt, bis man von allen Seiten vollständig darauf vorbereitet ist; denn ich sage Ihnen, nicht zu Tausenden, sondern zu Hunderttausenden werden die Kämpfenden in den bevorstehenden Schlachten fallen." 1

Im Original bildeten die vorstehenden, Hessens politische, sozioökonomische und militärische Weltanschauung in ihrer Geschlossenheit so trefflich erläuternden vier Abschnitte nur einen einzigen Paragraphen. Dergleichen im allgemeinen mit einem Land oder einem Gegenstand beschäftigte Paragraphen liefen ohne jegliche Untertitel von einer der riesenhaften Spalten - in Zoll gerechnet mass eine Seite vor $186628 \mathrm{r} / 2 \times 233 / 8$ und von da an bis Dezember $1869203 / 4 \times$ I 4 - bis zur folgenden. In der Regel bemühte eine einleitende Bemerkung oder ein Schluss sich darum, der ganzen Sache etwas Form zu verleihen. Vorankündigungen und Rückbezüge lieferten von Zeit zu Zeit eine Schnur, an der die einzelnen Berichte aufgereiht wurden. Indessen wurde keine Anstrengung sichtbar, ein literarisches Ganze zu schaffen ${ }^{2}$ oder die stellenweise recht mässige ${ }^{3}$ Qualität des Stils durch mehr als gelegentliche Geistesblitze zu heben. In dieser Beziehung ist die Leserschaft Chikagos offensichtlich selten verwöhnt worden.

Das hätte auch das europäische Publikum von sich sagen können,

1 Ibid., Bd. XXI (1867), Nr. 22, S. 3. Sperrung im Orig.

2 In einem Schreiben an Hess vom is. Dezember 1867 riet Hermann Raster davon ab, einen „laufenden Faden oder oft nur mühsam gefundene Übergänge" zu benutzen, da das dem amerikanischen Geschmack widerspreche.

${ }^{3}$ Gewisse Fehler scheinen Bildungslücken zu verraten, mögen aber auch bei der Herstellung der Zeitung entstanden sein. Hermann Raster bat in seinem Brief vom 18. Januar I 870 an Hess unter Anführung von Beispielen um weniger französiche Wörter. Die Schreibweise des im Exil Lebenden wirkt ab und zu leicht französiert. 
wäre es auf Hessens Kunst des Zeitungsschreibens angewiesen gewesen. Was er nach Amerika schickte, entsprach in solchem Grad seinen übrigen journalistischen Unternehmen, dass das Urteil seines Biographen bezüglich der Artikel über französische Angelegenheiten in den vierziger Jahren wie der späteren Beiträge zum "SozialDemokrat" sich ohne weiteres auf das Neugefundene ausdehnen lässt. ${ }^{1}$ In mindestens einem Fall enthielten die Briefe an das zuletzt genannte Blatt und die an die „Staats-Zeitung” sogat einen identischen Satz. ${ }^{2}$ Nach den Themen zu urteilen, mögen weitere Forschungen noch mehr Übereinstimmung in Stoff und Gedankengut zutagefördern. Letzteres änderte sich gelegentlich mit der wechselnden Flut „der augenblicklichen Situation und Volksstimmung" ${ }^{3}$, die getreu widerzuspiegeln nach Hess zur Aufgabe des Korrespondenten gehörte. Obschon gewiss nicht unübertroffen, zählt Hessens Leistung dennoch zu den wichtigeren Produkten des neunzehnten Jahrhunderts. Seine Analysen zeichneten sich, wie wir sehen, meist durch Gedankenreichtum aus. Reich war neben dem teilweise bereits behandelten Inhalt der Korrespondenzen die Auswahl der besprochenen Bücher, Broschüren, Zeitschriften und vor allem Zeitungen mit der dabeistehenden Erklärung ihres Standpunkts. Namentlich wer im fernen Amerika aus dieser höchst eindrucksvollen Presseschau ${ }^{4}$ einen Über-

1 Zlocisti, S. I00-101; 350-351.

${ }^{2}$ Cf. ibid., S. 352 und W.I.S.Z., Bd. XIX (1865), Nt. 43, S. I. Hess bezieht sich ausdrücklich auf solche Identität ibid. - Bandzahl verdruckt - Nr. 48, S. 2. Auszüge aus dem Sozial-Demokrat, ibid., Bd. XXIII (1 869), Nr. 34, S. 3.

${ }^{3}$ W.I.S.Z., Bd. XX (I866), Nr. 37, S. 5. Hessens fluktuierende Ansichten, doch auch die Kontinuität seiner Auffassungen liessen sich durch eine Heranziehung seiner Berichte für die Augsburger Allgemeine Zeitung im Jahr 1859 erhellen. Schon damals glaubte er, „dass das neue Régime, welches 1852 begann, dergleichen Hieb-und Stichwunden kaum länger ertragen kann" (Nr. 271, Sp. 4423). "Auch der Widerstreit der Elemente, auch der Kampf der Geister, der Irrthum selbst und die Lüge, welche zur chronischen Krankheit werden und in Verrücktheit übergehen können - auch sie haben ihre Gesetze und Schranken", erkannte er bei einer Gelegenheit (ibid., Nr. 288, Sp. 4704).

4 Die von uns studierte Artikelserie erwähnt: Accusateur Public, Aftonbladet, Arbeiterhalle, Avenir National, Beobachter, Breslauer Zeitung, Bulletin International, Centre Gauche, Chant du Vote, Charivari, Constitutionnel, Corsaire, Courrier de l'Intéricur, Courrier d'Orient, Courrier Français, Courrier Russe, Démocratie européenne, Demokratisches Wochenblatt, Der schwäbische Merkur, Diable-à-quatre, Eclipse, Egalité, Electeur Libre, Emancipation, Emancipation de Toulouse, Epoque, Etendard, Faubourg, Fédéraliste, Figaro, France, Gambetta, Gaulois, Gazetta Piemontese, Gazette de France, Gazette des Tribunaux, Germania, Gironde, Globe, Homme qui rit, Indépendance Belge, Indépendant de Parme, Indépendant du Centre, Indépendant du Tarne, International, Invalide Russe, Italia, Journal de l'Empire, Journal de Paris, Journal de Rome, JournaJ de Saint-Pétersbourg, Journal des Débats, Journal du Hâvre, Kölnische Zeitung, Lanterne, Liberté, Libre Pensée, Marseillaise, Mémorial Diplomatique, Monarchie Constitutionelle, Monde Clérical, Moniteur de l'Armée, Moniteur Universel, Morale Indépendante, Fortsetzung Fussnote auf S. 226. 
blick über die Vorgänge in Europa zu gewinnen versuchte, erhielt damit eine Gelegenheit, wie sie von fremdsprachigen Organen dort heutzutage kaum mehr geboten wird.

Die Staats-Zeitung selbst äusserte sich allerdings kritischer über ihren Pariser Vertreter. Dieser sagte schon 1866 voraus, „der erste Schuss in den Donaufürstenthümern... würde das Signal zum Ausbruch des europäischen Krieges sein". ${ }^{1}$ Doch sollte er den Weltkrieg und „die Stimme der deutschen Revolution, welche jene des Kanonendonners zum verstummen bringen könnte" ', nicht mehr erleben. Stattdessen sah er einen lokalisierten Konflikt, durch welchen sowohl das in seinen Wochenberichten bekämpfte System wie diese selbst beendet wurden. Die Ironie des Schicksals wollte es, dass die erste kritische Fussnote der Redaktion gegen ,alle diese Träume unseres Herrn Correpondenten" ${ }^{3}$ in dem Augenblick erschien, als dieser über die für ihn unzweifelhafte Mission des modernen Preussen, die zukünftigen Geschicke Deutschlands zu lenken, meditierte. „Ich bin der Ansicht, " war sein Kommentar zu dem in "musterhafter Organisation” durchgeführten Krieg gegen Österreich, „dass kein Grund mehr dazu vorhanden ist, an der Verwirklichung eines aufrichtig constitutionellen Preussens und Deutschlands zu zweifeln". ${ }^{4}$ Hessens schwärmerischer Bericht über den Genfer Friedenskongress vom September 1867 - ,die europäische Demokratie ist hier zu Wort gekommen und verdient es um so mehr gehört zu werden, als alle Anzeichen dafür sprechen, dass dieses Wort das Vorwort entscheidender Ereignisse ist" 5 - veranlasste das Blatt zu einem von diesem Optimismus sarkastisch abweichenden Leitartikel. ${ }^{6}$

Doch brach erst anlässlich der Endkrise des Kaiserreichs eine ernstere Unstimmigkeit zwischen Chikago und Paris aus. In der

Morgen-Post, Morning Chronicle, Moskauer Zeitung, Nain Jaune, Narodny-Listy, National de 1869 , National-Zeitung, Neue freie Presse, Niederrheinischer Courier, Nord, Norddeutsche Allgemeine Zeitung, Opinion Nationale, Opposition, Palais, Parlement, Patrie, Pays, Père Duchènes, Peuple, Peuple de Marseille, Peuple français, Phare de la Loire, Politica, Posener Zeitung, Presse, Presse Libre, Progrès de Lyon, Progrès Libéral, Punch, Quatre-vingt-treize, Radical, Rappel, Réforme, Renaissance, Revanche, Réveil, Révolution démocratique et sociale, Revue Contemporaine, Revue des deux mondes, Rumanal, Sächsische Zeitung, Satire, Sozial-Demokrat, Spenersche Zeitung, Standard, Tablettes, Temps, Times, Union, Vengeur, Voix de Proscrit, Voix du Peuple, Volksstaat, Vorbote, Weserzeitung, Wiener Arbeiterzeitung, Wiener Morgenpost, (Wiencr) Presse, Provinzial-Correspondenz, Zukunft.

1 W.I.S.Z., Bd. XX (1866), Nr. 23, S. 5. Sperrung im Orig.

2 Ibid., Nr, 22, S. 5 .

3 Ibid., Nr. 3 I, S. 4.

4 Ibid. Hess bedauerte "diesen Wahn" bald (ibid., Nr. 37, S. 5).

5 Ibid., Bd. XXI (1867), Nr. 39, S. 2.

${ }^{6}$ Ibid., S. 4. Der Artikel bezieht sich zweimal auf die Korrespondenz. 
Annahme, dass Napoleon erledigt war, schätzte Hess die Zahl der eine kaiserliche Massnahme missbilligenden Deputierten auf rund Ioo und fügte hinzu: „Kein Zweifel, dass diese die grosse Majorität des Landes und des ganzen intelligenten Frankreichs vertreten". ${ }^{1}$ In einer Klammer unterbrach der amerikanische Redakteur hier:

„Aber warum 'kein Zweifel'? Es ist eine sehr bequeme Art, sich das allgemeine Stimmrecht zurechtzulegen, wenn man stets ohne weiteres annimmt, dass die Minorität der Gewählten die Majorität der Wähler vertrete. Aber so rechnet man mit Wünschen, statt mit Wirklichkeiten. Man hat es den Bourbonen vorgeworfen, dass sie in zwanzig Jahren Nichts gelernt haben; aber die Herren in Paris haben auch in zwanzig Jahren nicht gelernt, was ausser ihnen jetzt alle Welt weiss, dass Paris nicht Frankreich ist. A. d. Red."?

Im selben Bericht wurde der Kommentar zu einem Tumult im Pariser Parlament, dadurch werde die neue (liberalere) Ära nicht befestigt, durch den Redaktionsvermerk erweitert, dadurch werde auch die parlamentarische Unfähigkeit der Franzosen bewiesen. ${ }^{3}$ Der Korrespondent wies den gegen ihn gerichteten Angriff in einem nachfolgenden Artikel unter Zitierung der Londoner "Times" zurück. ${ }^{4}$ Das hinderte die Redaktion nicht, ihre Kritik an der Wahlmathematik des Berichterstatters, dessen Parteilichkeit vom Standpunkt der amerikanischen Berichterstattung aus sie auch in einer anderen Sache durch eine Fussnote kritisierte ${ }^{5}$, in ironischen Worten zu wiederholen. ${ }^{6}$ Danach vergingen sieben Wochen, ohne dass ein Pariser Brief erschien, und hierauf folgte nur noch ein einziger der Serie, von der mehrere Stücke, wie aus der Numerierung hervorgeht, überhaupt nicht gebracht wurden.

Es ist kaum anzunehmen, dass äussere Umstände die Unterbrechung verursachten. Als zwei Wochen später der deutsch-französische Krieg ausbrach, pries der als Achtundvierziger gekommene Hermann Raster vor einer deutsch-amerikanischen Menge die Führungstolle der seit drei Jahren von ihm geleiteten Staats-Zeitung. „Er mahnte die Versammelten ernst, sich im Augenblick als Deutsche und Deutsche allein zu fühlen, als das Volk, dem die Zukunft Europas gehört, als das Volk, dessen Brüder über dem Ozean die Zukunft

1 Ibid., Bd. XXIV (870), Nr. 9, S. I.

2 Ibid. Sperrung im Orig.

3 Ibid.

4 Ibid. Nr. I6, S. 2.

5 Ibid., Nr. 5, S. 1 .

6 Ibid., Nr. 19, S. 2. 
Amerikas gehören wird". ${ }^{1}$ Ohne Rücksicht auf Hessens kürzliche Meinung, wonach Frankreich aus Ehrgefühl „zum revolutionärsten Lande der Welt" ${ }^{2}$ geworden (und damit zur Rettung berufen) sei, tadelte man in Chikago jetzt summarisch „die tohe gallische Räuberund Kehlabschneiderbande" für „den infamen Gewaltstreich des Franzosenkaisers gegen Deutschland". ${ }^{3}$ Nur ein Gefühl ziemte dem Deutschamerikaner: „Seine Faust ballt sich gegen den fränkischen Erbfeind". ${ }^{4}$ Die grosse teutonische Völkerfamilie vertrete seit Jahrhunderten "das Centrum der Civilisation" ${ }^{5}$, erfuhr man nun, ebenso, „dass fast alle Eigenschaften unabhängiger Männer, auf welche die Amerikaner am meisten stolz sind, deutschen Ursprung haben". 6 Dagegen musste „ein solches Barbarenvolk” wie die Franzosen „zum Heil der Menscheit für alle Zeiten unschädlich gemacht" 7 werden. Kurz, Chauvinismus regierte die Stunde.

Diese nach dem Zusammenbruch der kaiserlichen Diktatur fortgesetzte Haltung kontrastierte scharf mit der Hessens und seiner internationalistischen Freunde. ${ }^{8}$ Vor vier Jahren hatte der „Träumer” über die Zwangsläufigkeit eines möglicherweise unter einem preussischen Kaiser zu bildenden geeinten Deutschland geschrieben. Es werde einen Krieg hervorrufen, ,welche Regierung auch in Frankreich am Ruder sein möge". 9 Jetzt, wo all das Tatsache wurde, wandte er sich gegen „eine verfallene Nation” und verlangte eine „Koalition aller Völker"10 gegen das die französischen Freiheiten erstickende prussifizierte Deutschland. Das forderte er auch noch, nachdem er von Frankreich nach Belgien vertrieben worden war durch ,jene herrlichen 'internationalen Arbeiter', die gleich nach Verkündigung der Republik von deutscher Hiebe Gnaden die Verjagung aller Deutschen aus der Stadt durchgesetzt haben"11 (wie die "Staats-Zeitung” ohne Bezug auf den Mitarbeiter kommentierte). Die Ausweisungsmassnahme hatte Hess übrigens 1867 vorausgesagt. ${ }^{12}$

${ }^{1}$ Ibid., Nr. 29, S. 3. Siehe The Hermann Raster Papers, in: The Newberry Library Bulletin, Nr. 3 (I945), S. 24-29.

2 W.I.S.Z., Bd. XXIV (1870), Nr. 13, S. 2.

3 Aus zwei Artikeln in der Sonntagsausgabe der Staats-Zeitung, Der Westen, Bd. XVI (I 870 ), Nr. 29, S. 4 .

4 Ibid.

5 T.I.S.Z., Bd. XXIII (1870), Nr. 172 , S. 2.

${ }^{6}$ Ibid., Nr. I74, S. 2.

7 Ibid., Nr. 195, S. 2.

${ }^{8}$ Siehe ibid., Nr. 2 13, S. 2, wo eine Notiz über den einzigen deutschamerikanischen Joutnalisten erscheint, der für die französische Republik sei.

9 W.I.S.Z., Bd. XX (1866), Nr. 36, S. 2.

10 Siehe Zlocisti, S. 402-403.

11 T.I.S.Z., Bd. XXIII (1870), Nr. 213 , S. 2.

12 W.I.S.Z., Bd. XXI (1867), Nr. 40, S. 5. 
Politische Differenzen konnten andere Unstimmigkeiten nur verschlimmern. Es seien wegen ,der unsanften Manier in welcher der atlantische Telegraph nicht bloss Ihnen, sondern allen europäischen Correspondenten mitspielt, welche sich in ihren Berichten ausführlich mit der grossen Politik beschäftigen" - so schrieb Raster an Hess in einem Brief vom I 5. Dezember I867 - „seit Monaten... kaum zwei oder drei Ihrer Briefe nicht mit den Kabelnachrichten überzwerch angekommen". ${ }^{1}$ Feuilletons brauchte er. Am I8. Januar I 870 wiederholte Raster, dass "politische Berichte nicbt sind, was wir brauchen". ${ }^{2}$ Das Interesse an Frankreichs Geschicken sei „sehr gering” und die telegraphisch übermittelten Tatsachen brächen aller Spekulation die Spitze ab. „Politische Vorausverkündigungen haben in 9 unter so Fällen gar keinen Werth". ${ }^{3}$ Nicht nach Zeitungsberichten, sondern nach eigener Anschauung sollte geschrieben werden. „Das Verhältnis von dem, was wir brauchen zu dem was Ihre Berichte sind, ist das von Farbendrucken zu Bleistift-Planzeichnungen". "Am I9. März schliesslich klagte Raster, dass seine Mahnungen ,gänzlich unberücksichtigt zu bleiben scheinen. Politik, Politik, und immer weiter Politik... für bier völlig ungeniessbar". ${ }^{5}$

Widerstrebe es seinen Neigungen, Feuilletonistisches zu liefern (schloss der Redakteur seine Epistel an den Abgekanzelten), dann möge er seine ,politischen Mittheilungen in höchstens zwei Briefen per Monat" " 6 geben; man werde alsdann einen neuen Mitarbeiter zu gewinnen versuchen. Drei Monate darauf, am 28. Juni 1870 , erschien (soweit zu sehen ist) Hessens letzter Bericht. Er war rein politisch gehalten und besonders trocken. Unmittelbar danach begann Bruno Bauer ", den Raster beinahe in den gleichen Ausdrücken, die er für Hess verwandte, darum bat, gegen ein Honorar von fünf Dollar pro Brief zweiwöchentliche Schilderungen zu liefern ${ }^{8}$, seine Mitarbeit von Berlin. Am 5. August konnte das Blatt dann in einer an Deutlichkeit nichts $\mathrm{zu}$ wünschen übriglassenden Mitteilung verkünden: „Wir veröffentlichen heute den ersten einer Reihe von Correspondenzberichten aus Deutschland, welche Friedrich Kapp der Ill. Staatsztg. liefern wird. In Kapp haben wir endlich einen solchen Berichterstatter für unsere Zeitung gefunden, wie wir ihn uns lange gewünscht haben, d.h. einen solchen, der, mit den Anschauungen und Bedürf-

1 Unterstrichen im Orig. Der Briefschreiber gibt Beispiele für das Nachhinken der Berichte.

2 Unterstrichen im Orig.

3 Ibid. Es folgen wieder Beispiele.

4 Ibid.

${ }^{5}$ Brief an Hess.

- Ibid. Unterstrichen im Orig.

7 W.I.S.Z., Bd. XXIV (1870), Nr. 88, S. I.

${ }^{8}$ Siche oben, S. I, Fussnote 3). 
nissen des deutsch-amerikanischen Lesepublikums vollkommen vertraut, sich nicht versucht fühlen wird, seine Berichte mit solchen Mitttheilungen auszufüllen, welche hier aus europäischen Zeitungen oder Telegrammen längst bekannt sind und daher allen Wert verloren haben".

Doch gab es noch einen anderen Epilog. Wenige Jahre nach seiner Rückkehr in die französische Hauptstadt starb Hess fast gleichzeitig mit Herwegh, dem Poeten und Politiker, mit dem er vor einer Dekade dem Chikagoer Publikum vorgestellt worden war. ${ }^{2}$ Nur den letzteren ehrte die Staats-Zeitung mit einem Nachruf. ${ }^{3}$ Zwei Wochen nach dem Hinscheiden des freiheitlichen Dichters aber bedauerte sie in einem Leitartikel die Unterwürfigkeit der deutschen Presse: „Die Deutschen im Auslande haben vor vier Jahren den Siegen ihrer Stammesgenossen über den 'wälschen Erbfeind' freudig zugejauchzt und sich über die Grösse, Macht und Herrlichkeit des neuen deutschen Reiches bass begeistert. Doch ist, was drüben geschieht, wahrlich nicht dazu angethan, dieser Begeisterung Nahrung zu geben". ${ }^{4}$ Der Schlussstein des Reichsgebäudes, meinte der recht scharfe Artikel, hätte nicht eine vergängliche Person, sondern die Freiheit sein müssen. Gerade das aber war Hessens Standpunkt. „Nur die Freiheit,” schlug er einst für ein Problem vor, das auch wir noch nicht gelöst haben - das des Nahen Ostens - ,ist hier, wie überall, die definitive Lösung”. ${ }^{5}$

1 T.I.S.Z., Bd. XXIII (1 870), Nr. 1 82, S. 2. In ihrer Arbeit Friedrich Kapp, Leipzig 1935, gibt Edith Lenel eine ausgezeichnete Darstellung ihres Vorfahren, allerdings ohne Benutzung der Staats-Zeitung.

${ }^{2}$ Siehe oben, S. 4.

3 W.I.S.Z., Bd. XXIV (1875), Nr. 17, S. 5.

4 Ibid., Nr. I6, S. 4 .

5 Ibid., Bd. XXIII (1869), Nr. 4, S. 2. 